\title{
Just the Facts: Academic Press FactsBooks on Gene Knockouts and Oncogenes/Tumor Suppressors
}

\author{
A Oberst \\ Cell Death and Differentiation Editorial Office; Correspondence to drew_oberst@hotmail.com \\ doi:10.1038/sj.cdd.4401052
}

The Oncogene and Tumor Suppressor Gene FactsBook, 2nd edition. By R Hesketh. Academic Press, San Diego, CA: 1997. Pp 549. ISBN: 0-12-344548-5. £37.95 Sterling

The Gene Knockout FactsBook. Edited by TW Mak. Academic Press, San Diego, CA: 1998. Pp 1140. ISBN: 0-12-466044-4 (2 volume set). £46.95 Sterling

There are an awful lot of biologists in the world, and they've discovered (and knocked out) an awful lot of genes. Add to this the fact that the papers describing these genes are spread over thousands of journals, and it's enough to make you want to throw up your hands and try something easy, like land mine removal. With around 60000 functional genes in humans alone, most of us have, at some point, come across a gene name and wondered 'What the dickens is that? What does it do? And what happens when it's removed?' The folks at Academic Press have addressed the rapid pace and widely dispersed nature of biological publication by condensing the salient points on a variety of topics into aptly named FactsBooks. FactsBooks are currently available dealing with all sorts of functional protein families, from nuclear receptors to adhesion molecules, and ion channels to G-linked proteins. We chose to take a look at the 'Gene Knockout FactsBook' and the 'Oncogene Tumor Suppressor Gene FactsBook' (2nd Edition).

The 'Gene Knockout FactsBook' is, not surprisingly, a no-nonsense guide to gene knockouts in the mouse. The 2-volume set is divided into two rather uneven sections: a 4-page 'Section l' provides a brief but thorough introduction, while the 1131-page 'Section II' provides 1-2-page summaries of about 600 different gene knockouts. The introduction provides a brief background on the techniques and pitfalls of genetic knockouts, including brief discussions of homologous recombination, embryonic stem cells and their uses in transgenics, the neo ${ }^{r}$ system, generation of point mutations, and the difficulties associated with controlling genetic background. Having summarized these topics, the book plunges headlong into the individual knockout genes. Each entry lists alternative names for the gene of interest, its symbol/abbreviation, accession number, and general 'area of impact' (e.g. development, metabolism, immunity etc.) Several lines are then dedicated to a 'General Description', which outlines the function of the gene of interest including its superfamily, expression pattern, and interaction partners. A description of one or more KO strain construction strategies follows, along with a description of the phenotype of the knockout, general comments, and several references for further reading.
Let's take the fas gene as a case study. The FactsBook notes that fas is also known as CD95 and APO-1, and cites its area of impact as 'apoptosis'. It acknowledges that the Fas protein belongs to the TNF receptor superfamily, comprises 306 amino acids, is expressed ubiquitously with particular abundance in the heart, liver, and thymus, and that Fas is capable of activating the caspases. It goes on to describe KO strain construction: exon 9 was replaced with a DNA fragment carrying neomycin resistance, ES cells carrying the desired mutation were then injected into blastocysts, and the genotype was confirmed by the lack of Fas mRNA, by immunoprecipitation of thymocyte cell surface proteins, and by flow cytometry (references included). The Fas $-/-$ phenotype is then described, including fertility (normal) and survival (5 months). The finding that the lymph nodes and spleen of Fas - / - mice were about 40 and 10 times larger than normal, respectively, at 16 weeks of age is mentioned, as is the increased presence of blood lymphocytes. Abnormal immune system and liver development are also described. These observations are summarized in the 'Comments' section with a note that Fas is involved in clonal deletion of activated mature Tcells, as well as B-cells and possibly senescent hepatocytes. Two references, dating from 1995 and 1996, are provided.

'The Oncogene and Tumor Suppressor Gene FactsBook', by contrast, has a narrower area of focus, and therefore provides more in-depth information; it reads slightly more like a textbook rather than a straight reference volume. The tome kicks off with a 66-page introduction surveying cancer development. This well-written, illustrated, and referenced summary covers the mechanisms of oncogene activation, transforming alterations in the proliferation control pathways, tumor suppressor function and deactivation, DNA repair function and defects, and metastasis-in short, it summarizes the major points in our current understanding of oncogenesis. This introduction is followed by about 20 pages of tables, which provide both a quick reference and sub-division of genes by common characteristics, e.g. 'oncogenes activated by retroviral insertion,' or 'tumor suppressor genes detected in human tumors'. All these tables are very well referenced, providing 
instant bibliographical information on the defining studies of virtually any gene of interest.

Following the 'Tables' section, the book moves into the reference section; the majority of the remainder is dedicated to 59 entries on oncogenes, though there are also 15 tumor supressors, 4 CDK inhibitors, and two types of DNA tumor viruses discussed. Unlike the 1-2 page entries in the 'Gene Knockout FactsBook', each entry is 510 pages long, and goes into significantly more depth. Let's look at the entry for p53: The original identification of p53 from SV40-transformed large-T immunoprecipitates is mentioned, as are vital stats like gene size, chromosomal location, protein mass, cellular location and tissue distribution. The function of the protein is then described in detail, with tables of 'Factors enhancing p53 binding to DNA', 'Sequences to which p53 binds', 'Genes repressed by p53', 'Kinases phosphorylating p53', and 'proteins associating with p53'. The roles of p53 in the normal growth cycle, apoptosis, and cancer are addressed in fair detail. The gene and protein structures are then discussed, with figures of each, and known functional mutations of p53 are listed, along with their common effects in vivo. The full amino acid sequence of p53 is then given, with key functional domains (in this case the $\mathrm{Zn}^{2+}$ binding domain) underlined, and a domain-by-domain breakdown of the sequence provided. This is followed by fully 255 references; though the importance of $p 53$ to human cancers means that this entry is slightly longer than some, the level of detail found in other entries is comparable.

In the final analysis, both these reference works perform their tasks well. Indeed, a major challenge in writing this review was keeping the books on my desk; it seemed that everyone who saw them wanted to take a closer look. The 'Gene Knockout FactsBook' provides key information about a large number of knockout genes, and would probably find a useful niche in a lab conducting knockout experiments, or indeed on the desk of anyone trying to keep up with the field. Though it does not go into great detail on any one gene, this volume covers a lot of ground, and is well enough referenced to act as a stepping stone for further investigation. 'The Oncogene and Tumor Suppressor Gene FactsBook', on the other hand, provides much more in-depth information on a smaller total number of genes, and with its excellent introduction provides the reader with all the tools necessary to come to grips with the perennially 'hot' field of cancer research. The superb referencing, and the wide variety of ways in which the information is presented (explanations, tables, figures, summaries) makes it easy to take what you read and run with it. I really can't think of a better or more thorough way to present this information. Both of these references do suffer from a significant shortcoming, though: Age. 'The Gene Knockout FactsBook' was published in 1998, and 'The Oncogene and Tumor Suppressor Gene FactsBook' dates from way back in 1997; both books are starting to show their age. In both cases, the majority of referenced papers are from the 1993-1996 period, and while these were reasonably up to date when the books were published and still represent seminal works in their respective fields, many new and relevant discoveries have taken place in the intervening years. However, recent refinements notwithstanding, the core information presented in both references remains extremely relevant, and I would consider these volumes a solid investment for any lab or scientist interested in the field. 